\title{
EXPERIENCES ET STRATEGIES DE FEMMES INVESTIES DANS UN “MONDE D'HOMMES” Le cas de la politique locale portugaise
}

\author{
Maria Helena Santos \\ Instituto Universitário de Lisboa (ISCTE-IUL), CIS-IUL, Lisbonne, Portugal, et Centre en Etudes \\ Genre LIEGE, Université de Lausanne, Lausanne, Suisse
}

Patrícia Roux

Revue Nouvelles Questions Féministes, Lausanne, Suisse

Lígia Amâncio

Instituto Universitário de Lisboa (ISCTE-IUL), CIS-IUL, Lisbonne, Portugal

Résumé L'article présente une recherche sur les dynamiques de genre œuvrant dans un contexte de tokenism: la politique. Vingt-deux femmes politiques portugaises ont été interviewées. Leurs stratégies pour se faire une place dans le monde masculin de la politique sont surtout déterminées par l'âge: les plus âgées luttent contre les discriminations de genre et privilégient des compétences féminines, tandis que les plus jeunes valorisent le profil de la superwoman qui se distancie de son groupe socialement dominé, au risque de dénier la discrimination vécue, et se montrent très attachées à l'idéologie méritocratique menacée par les quotas.

Mots-clés: tokenism, politique, genre, discrimination, discours.

Resumo O artigo apresenta uma investigação sobre as dinâmicas de género que operam num contexto de tokenism: a política. Vinte e duas mulheres políticas portuguesas foram entrevistadas. As suas estratégias para assegurar um lugar no mundo masculino da política são sobretudo determinadas pela idade: as mais velhas lutam contra as discriminações de género e privilegiam as competências femininas, enquanto as mais jovens valorizam o perfil da superwoman, que se distancia do seu grupo socialmente dominado, correndo o risco de negar a discriminação experimentada, e mostram-se muito ligadas à ideologia meritocrática ameaçada pelas quotas.

Palavras-chave: tokenism, política, género, discriminação, discursos.

Abstract The article presents a research on the gender dynamics operating in a tokenism context: politics. Twenty-two Portuguese female politicians were interviewed. Their strategies to get a place in the masculine world of politics is mainly determined by the age: whereas the older women fight against gender discriminations and favor women's skills, the younger value the superwoman profile that distance themselves from their socially dominated group, at the risk of denying the experienced discrimination, and are very attached to the meritocratic ideology threatened by the quotas.

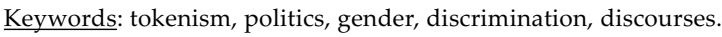

Resumen El artículo presenta una investigación sobre las dinámicas de género que operan en un contexto de tokenism: la política. Veintidós mujeres políticas portuguesas fueron entrevistadas. Sus estrategias para hacer su lugar en el mundo masculino de la política están principalmente determinadas por la edad: las de más edad luchan contra las discriminaciones de género y favorecen las competencias femeninas, mientras que las más jóvenes favorecen el perfil de la superwoman, que se distancia de su grupo socialmente dominado, al riesgo de negar la discriminación experimentada, y se muestran muy apegadas a la ideología meritocrática amenazada por las cuotas.

Palavras-clave: tokenism, política, género, discriminación, discursos. 


\section{Introduction}

La sous-représentation des femmes en politique est un phénomène international (Krook et O'Brien, 2010; Gaspard, 2007). Face à cette inégalité, plus d'une centaine de pays se sont dotés de quotas électoraux et de lois sur la parité (Dahlerup, 2008). De telles mesures ont contribué à féminiser la profession politique, mais elle continue à être structurée par la division sexuelle du travail et la féminisation ne s'accompagne pas d'un réel partage du pouvoir entre hommes et femmes politiques (Sineau, 2011). Nous considérons donc que les professionnelles de la politique sont en position de "token" (Wright, 2001), c'est-à-dire constituent une minorité non seulement parce qu'elles sont moins nombreuses que les hommes dans le champ, mais surtout du fait que la politique reste un monde que la classe des hommes domine et dans lequel elles doivent lutter pour légitimer leur place. A partir de ce point de vue, le présent article s'attache à cerner les expériences de femmes travaillant dans le monde masculin de la politique et les stratégies de genre qu'elles développent dans ce contexte. Notre objectif principal est de voir de quelles manières elles gèrent leur statut doublement minoritaire et tentent de s'émanciper aussi bien de leur statut de minorité dans le monde politique que de leur statut de groupe socialement dominé dans les rapports sociaux de sexe.

Le terrain de l'analyse proposée se situe au Portugal, qui a introduit une Loi sur la parité en 2006, et l'étude porte sur la politique locale parce que les résistances au changement y sont particulièrement visibles (Santos, 2011; Santos et Amâncio, 2012). Dans un premier temps, l'article présente le cadrage théorique de l'étude que nous avons menée, puis les résultats de celle-ci, dans laquelle 22 femmes engagées dans la politique locale ont été interviewées.

\section{Le tokenism: de l'analyse neutre à l'analyse de genre}

Le tokenism fait référence à tous les domaines dont l'accès est limité à certains groupes sociaux et, selon Wright (2001), il affecte trois groupes: le groupe dominé, le groupe dominant et les tokens. Dans notre étude, ces trois groupes sont respectivement les femmes, les hommes et les élues. Dans l'étude princeps de Kanter en entreprise (1993 [1977]), trois raisons conduisent l'auteure à considérer que les tokens sont une minorité numérique désavantagée et discriminée par rapport au groupe majoritaire: ils sont particulièrement visibles, et ainsi mis sous pression pour être performants; les différences entre les tokens et les dominants sont polarisées, pouvant conduire à la marginalisation des premiers; ils sont enfermés dans des rôles stéréotypiques associés à leur groupe d'appartenance dominé.

Ce travail de Kanter a été critiqué du fait qu'elle n'a retenu qu'un critère numérique pour définir les groupes, alors que de nombreux autres facteurs - structurels, culturels, sociaux et psychologiques - ont aussi un impact sur leurs interactions (voir Santos et Amâncio, 2014). Notamment, les dynamiques du tokenism qu'elle a mises au jour ne prennent véritablement leur sens qu'à la condition de prendre en compte que les individus qu'elle analyse sont des femmes et de 
replacer ces dynamiques dans les rapports de genre (Yoder, 1994). La relation hiérarchique entre les deux catégories de sexe détermine en effet la nature des expériences que font les tokens et, par exemple, leur mobilité professionnelle: les hommes sont généralement favorisés par un "escalier roulant ascendant" (Williams, 1995) lorsqu'ils s'investissent dans un groupe professionnel féminin, comme dans certains secteures de la médecine où les femmes sont désormais en nette majorité (Santos, Amâncio et Roux, 2015), tandis que celles-ci se heurtent à un "plafond de verre" qui les maintient écartées des postes de pouvoir dans les métiers masculins (e.g., Cognard-Black, 2004; Floge et Merrill, 1986; Heikes, 1991; Ott, 1989), y compris dans le domaine politique (e.g., Bereni et al., 2011).

Dans ces situations inégalitaires, les femmes se sentent souvent sous pression, voire menacées parce qu'elles drainent derrière elles l'histoire d'un groupe historiquement défavorisé et dominé (Barreto, Ellemers et Palacios, 2004). C'est le cas lorsqu'elles sont soupçonnées d'être incompétentes et pas à leur place dans un "métier d'homme". Les travaux de Santos (2011), poursuivis dans notre étude présentée ici, montrent que les femmes - des élues en l'occurrence - sont alors obligées de prouver qu'elles sont compétentes et résistantes, leur présence ne va jamais de soi. Et pour peu qu'elles se comportent de façon contre-stéréotypique (e.g., sont ambitieuses, audacieuses, autoritaires), elles courent le risque d'être stigmatisées (Heilman et al., 2004).

L'intégration des femmes dans des métiers masculins se fait dans un cadre idéologique particulièrement propice à masquer les logiques institutionnelles inégalitaires, celui de l'idéologie méritocratique. En effet, différentes études ont montré que l'adhésion des tokens à cette idéologie peut les amener à ignorer les discriminations subies par les groupes dominés (Ellemers, Spears et Doosje, 1997) et à défendre le statu quo qui leur a permis d'obtenir une position supérieure (Barreto, Ellemers et Palacios, 2004). D'autres travaux établissent que les discriminations contre "les femmes en général" sont reconnues, mais que ce sont les discriminations vécues personnellement qui sont déniées (e.g., Crosby, 1984; Roux, 1999). Les femmes elles-mêmes auraient tendance à se "détacher" de leur groupe de sexe pour maintenir la culture organisationnelle qui a favorisé leur succès (Apfelbaum, 1999), voire se désolidariseraient de leur groupe en privilégiant des discours individualistes qui les placent dans une position méritante et qui placent en revanche leurs pairs dans une position de non-méritantes. Ainsi, le tokenism semble entretenir l'idéologie du mérite (Taylor et McKirnan, 1984), soutenir les mythes légitimateurs de l'ordre social hiérarchique délimitant "qui a droit à quoi?" (Staerklé et al., 2007), et constituer un phénomène institutionnalisé qui maintient les privilèges des hommes et leur position de classe dominante (Williams, 1995). Si l'on y ajoute les difficultés, évoquées plus haut, que les femmes rencontrent lorsqu'elles s'engagent dans un métier masculin, l'on peut penser que leur présence dans ce type d'espaces sert plus d'alibi que de moteur d'un changement des pratiques et des normes de genre. 


\section{Genre, tokenism et action positive: le cas des femmes politiques}

Parmi toutes les controverses qu'a générées la question des quotas électoraux et de la parité en politique (Lépinard, 2007; Scott, 2005), l'argument du mérite continue à tenir une place prépondérante dans les discours des politiciens et du corps électoral: le mérite est une règle de justice prédominante dans nos sociétés (Young, 1990) que ces discours estiment contournée par les lois sur la parité ou les quotas (Santos, Amâncio et Alves, 2013). Cet argument insuffle l'idée que l'inclusion des femmes dans les listes électorales par les quotas péjorerait la qualité du travail politique, soit le niveau de compétence. En d'autres termes, le mérite des femmes politiques est mis en question, même si l'argument ne tient nullement: prétendre à une violation du mérite suppose qu'il peut être objectivement mesuré (Crosby et Clayton, 2001), or l'on sait que l'évaluation du mérite n'est pas "neutre" (Young, 1990). Celui des hommes politiques par exemple est généralement peu questionné (Gaspard, Servan-Schreiber et Le Gall, 1992). Dans le métier politique comme dans les autres métiers masculins, l'accès au mérite fait donc partie des dynamiques du tokenism qui figent les rapports sociaux hiérarchiques et entretiennent les résistances aux transformations des pratiques et des règles de genre inégalitaires (Santos, 2011).

$\mathrm{Au}$ Portugal, ces résistances sont particulièrement visibles au niveau de la politique locale (Santos et Amâncio, 2012), portées surtout par les hommes, ceux-ci se sentant menacés par la mise en œuvre de la Loi sur la parité (Lei Orgânica n. $.3 / 2006$ ). Plus largement, c'est l'ensemble des mesures d'action positive promouvant l'égalité des sexes au sein du monde politique qui sont plus appuyées par les femmes. Si le soutien des femmes et des hommes à ces mesures se différencie, c'est bien entendu en raison de l'inégalité de leurs positions respectives dans les partis politiques, mais aussi dans la vie privée, car même dans les professions prestigieuses (Fassa et Kradolfer, 2010) parmi lesquelles nous incluons "être femme politique" (Sineau, 2011), le travail domestique continue d'être de la responsabilité principale des femmes (e.g., voir Amâncio, 2007; Geist, 2010; Silva, Jorge et Queiroz, 2012; Wall et Guerreiro, 2005).

C'est donc à partir des expériences familiales et professionnelles d'élues que nous avons étudié les effets du tokenism en politique. Notre étude vise à analyser les stratégies que des femmes engagées dans la politique locale portugaise développent pour s'affranchir de leur position doublement dominée, que ces stratégies confortent le système de genre ou au contraire œuvrent en faveur de l'égalité des sexes. L'intérêt de la démarche est qu'elle se situe dans un contexte de tokenism particulier: celui d'un espace politique dans lequel la Loi sur la parité a été appliquée.

\section{Méthode \\ Participantes}

Vingt-deux femmes des cinq partis en activité au niveau local ont participé à cette étude, menée au Centre (16 communes de Lisbonne et des environs) et au Nord (six 
communes de Porto et environs) du Portugal. Nous avons interviewé deux femmes du Bloc de gauche (BE), quatre du Parti communiste portugais (PCP) et six du Parti socialiste (PS), huit du Parti social-démocrate (PSD) et deux du Parti du centre démocratique et social - Parti populaire (CDS-PP). Quatorze exercent leur activité au niveau exécutif (une présidente d'une mairie, sept présidentes de petites communes et six conseillères municipales) et huit au niveau délibératif (députées de différentes Assemblées municipales et communales). Seize sont mariées, deux sont divorcées, une est veuve et trois sont célibataires. Dix-sept ont des enfants, deux femmes mariées et les trois célibataires n'en ont pas. Onze femmes ont entre 32 et 53 ans $(M=42$ ans), tandis que l'âge des 11 autres varie de 54 à 78 ans $(M=64$ ans). Dans ce second groupe d'âge, l'activité politique des élues s'étend en moyenne sur près de 26 ans (médiane $=29$ ans), l'exercice de cette activité étant évidemment plus court, bien que solide, dans le groupe des plus jeunes $(M=16$ ans, médiane $=11)$. Ces dernières sont mieux représentées dans les partis de gauche (les deux-tiers des 12 élues de gauche ont entre 32 et 53 ans) et les interviewées plus âgées sont plus investies dans les partis de droite ( 7 des 10 élues de droite ont entre 54 et 78 ans).

\section{Procédure et dimensions thématiques}

L'échantillon des élues a été constitué avec la technique dite "boule de neige", en partant de nos contacts avec des députées du Parlement national. Sollicitées par courrier électronique, les 22 femmes politiques ont été interviewées entre avril et juin 2012, durant $1 \mathrm{~h} 30$ en moyenne. Les entretiens ont été enregistrés puis retranscrits intégralement, tout en garantissant aux élues leur anonymat.

Notre guide d'entretien semi-structuré englobait plusieurs questions divisées en six dimensions thématiques: les expériences des participantes dans l'exercice de leur métier politique (donc les dynamiques du tokenism); les compétences qu'elles jugent nécessaires pour "faire de la politique" de façon efficace; la conciliation des vies politique et familiale; les causes de l'inégalité des sexes dans la politique, leurs opinions sur la situation et sur des mesures telles que les quotas et la Loi sur la parité; leur sentiment sur la discrimination des femmes en général et d'elles-mêmes en particulier; leurs formes de mobilisation (individuelle ou collective) pour l'égalité des sexes.

\section{Procédure analytique}

Le corpus des 22 entretiens a été soumis à une analyse des données textuelles effectuée avec le logiciel Alceste 2012. ${ }^{1}$ Nous avons opté pour une procédure qui ne se centre pas sur la spécificité interne de chaque interview et donc sur la logique des discours ou des parcours individuels, mais qui vise à saisir la diversité ou la communauté des points de vue exprimés sur chacune des six dimensions thématiques décrites ci-dessus. Dans nos résultats, les dimensions seront désignées par les mots-clés

$1 \quad$ Nous avons utilisé la méthode de "classification descendante hiérarchique" que propose Alceste: à partir des (co)occurrences de mots et d'un découpage des discours en "unités de contexte", le logiciel crée des classes thématiques qui permettent d'identifier l'organisation des discours. 
suivants: (i) dynamiques, (ii) compétences, (iii) conciliation, (iv) in/égalité, (v) discrimination et (vi) mobilisation.

De manière à saisir l'ancrage social des classes identifiées par Alceste, nous avons projeté sur celles-ci trois variables sociodémographiques des interviewées: le statut marital (vit en couple $v$ s. seule), le nombre d'enfants (avec $v$ s. sans enfants) et le groupe d'âge construit à partir de l'âge médian ( 11 femmes de 32 à 53 ans vs. 11 de 54 à 78 ans). Une quatrième variable d'ancrage social a été considérée, l'appartenance politique, à partir d'une division dichotomique: gauche (BE, PCP, PS: 12 élues) vs. droite (PSD, CDS-PP: 10 élues). ${ }^{2}$

\section{Résultats}

Les résultats ont révélé une structure thématique divisée en deux grands groupes de cinq classes. Le premier groupe, incluant les classes 1, 2 et 3, est plus centré sur l'activité politique, tandis que le deuxième groupe, incluant les classes 4 et 5, intègre davantage d'éléments de la sphère privée, articulés à l'engagement des femmes dans la sphère publique. Nous allons nommer et interpréter ces classes en tenant compte des mots à partir desquels elles ont été construites et des "unités de contexte élémentaires" (les UCE sont des extraits d'interviews) qui les caractérisent (voir figure 1). ${ }^{3}$

\section{Dynamiques de genre faisant obstacle à la Loi sur la parité}

La classe 1 représente 10\% des UCE classées. Les discours qui ont le plus contribué à la formation de cette classe sont liés aux questions sur l'in/égalité, portés par l'ensemble de la population étudiée mais davantage encore par les élues de gauche et la catégorie d'âge la plus jeune (32-53 ans). ${ }^{4}$

Dans cette classe, nous avons identifié quatre thèmes organisant les prises de position des interviewées concernant la Loi sur la parité et les quotas: la division sexuelle du travail, le principe du mérite, les résistances des hommes et la minimisation de la discrimination personnelle.

Le premier thème est constitué par la division sexuelle du travail qu'elles voient comme un obstacle à la mise en œuvre des mesures paritaires, et ceci indépendamment du fait qu'elles sont favorables ou défavorables aux quotas. Comme l'illustre l'extrait suivant, les interviewées estiment que le monde politique n'est pas capable de s'organiser sur un mode égalitaire et que la difficulté, pour une femme, d'accéder au métier politique est renforcée par sa charge de travail dans la sphère domestique:

2 La dispersion des 22 interviewées entre les cinq partis rendait délicate une analyse effectuée au sein de chaque parti.

3 Alceste a classé $74 \%$ du corpus, ce qui constitue un bon indice de pertinence.

4 Le fait qu'un groupe d'interviewées soit plus représenté dans une classe ne signifie pas que les discours des autres élues ne contribuent pas à la formation de la classe. 


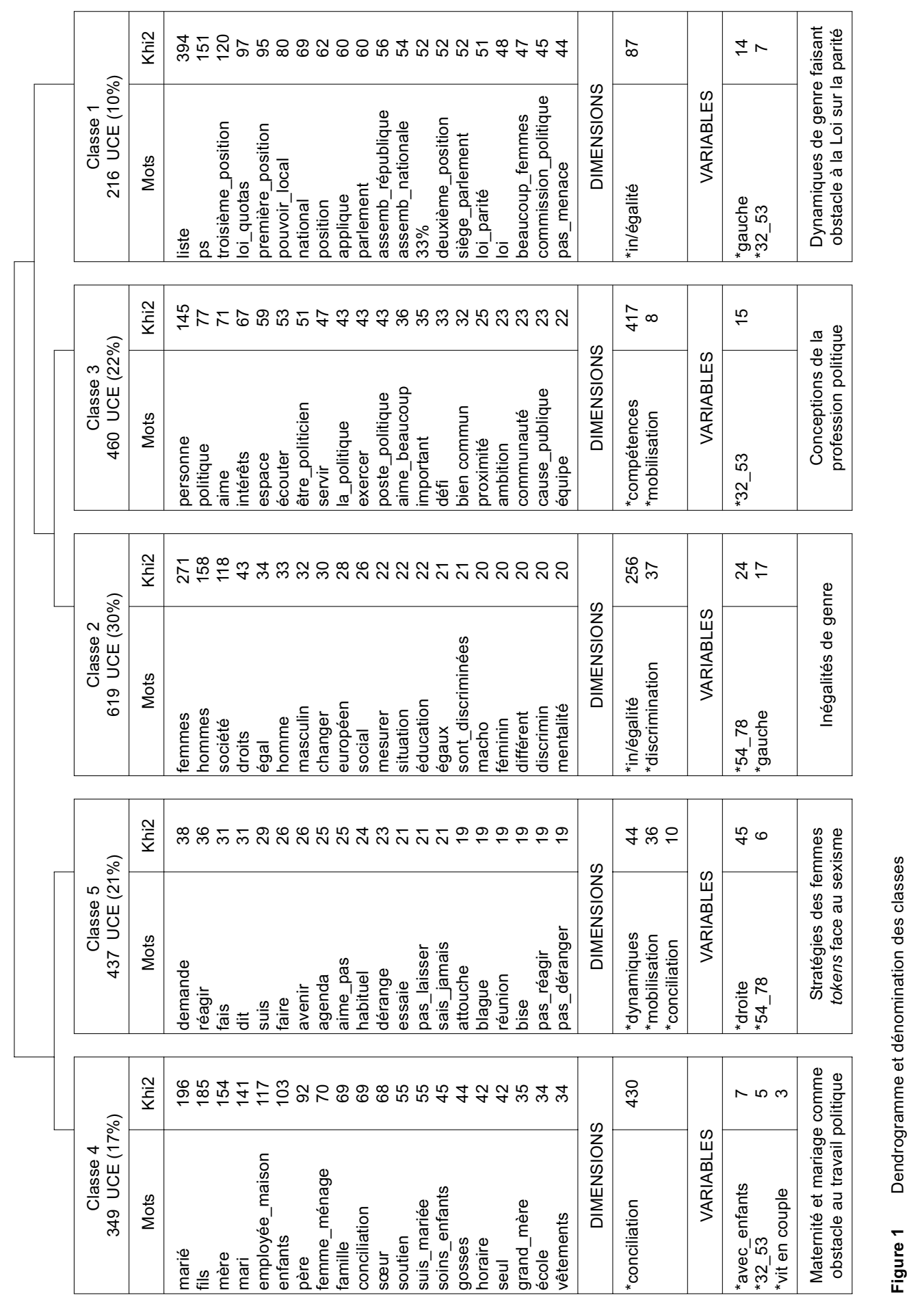


Les femmes ont de nombreuses contraintes, ont beaucoup de difficulté à faire partie d'une liste, d'autant plus qu'après, à mon avis, elles n'auront pas le temps de pouvoir suivre. [Présidente d'une commune, gauche (PCP), 32 ans, mariée, 1 enfant petit]

Une autre dynamique de genre qui fait obstacle à la mise en œuvre de la Loi sur la parité tient au poids de l'idéologie méritocratique. L'ensemble des participantes, mais avant tout les plus jeunes positionnées à gauche, estiment que l'égalité des sexes n'est pas respectée si le principe du mérite n'est pas lui-même respecté. Concrètement, le mérite se mesure aux efforts de la personne, à son investissement, à ses qualités et compétences. L'on relèvera cependant que seul le mérite des femmes est évalué, celui des hommes politiques va de soi. Ainsi, lorsqu'une femme est invitée à rejoindre une liste, ce doit être parce qu'elle est compétente, et non pas pour faire nombre, pour donner une bonne image du parti ou pour appliquer la loi, car sinon, cela serait injuste pour les hommes:

Les quotas, c'est très injuste parce que ce que j'ai toujours vu, c'est qu'on finit par remplir les listes avec des femmes qui étaient très peu impliquées auparavant, et que les hommes sont placés comme suppléants, les malheureux, alors qu'ils sont très compétents et se sont beaucoup donnés pour la cause. [Députée d'une Assemblée municipale, gauche (PS), 32 ans, mariée, 1 enfant petit]

Si l'idéologie méritocratique imprègne les conceptions de ces élues, c'est aussi parce qu'elles savent que les hommes et les partis résistent à l'entrée des femmes en politique et cherchent à préserver leurs privilèges masculins. En effet, elles font état des stratégies que les partis, y compris le PS - qui a impulsé l'idée des quotas -, ont développées ou développent encore pour détourner le sens premier, donc l'objectif d'égalité, des quotas ou de la Loi sur la parité. Les militantes se montrent relativement fatalistes, voire désabusées face à ces stratégies de résistance masculines, telles que répartir les femmes dans les listes de manière à ce qu'elles ne soient pas élues, nommer des femmes-alibis, ou encore augmenter le nombre de sièges pour que les hommes n'en perdent pas un au moment où les femmes politiques en conquièrent quelques-uns, comme l'exprime clairement cette élue:

Comme les hommes ne voulaient pas partir, la première fois que le quota des $25 \%$ devait être appliqué, Guterres [ancien premier ministre] n'a changé qu'une seule chose: il a augmenté de $25 \%$ le nombre de sièges dans les organismes nationaux, ce qui prouve que le gros problème, jamais explicite, est que les hommes ne veulent pas sortir. [Conseillère municipale, gauche (PS), 65 ans, mariée, 3 enfants adultes]

Ainsi, d'un côté, les interviewées dénoncent les fortes résistances des hommes et des partis à l'application de la parité. Mais de l'autre, elles se montrent très attachées au principe du mérite individuel. Or, aux yeux de beaucoup, ce principe est incompatible avec des mesures telles que les quotas, qui cherchent à corriger les positions inégalitaires des groupes sociaux. Prises en quelque sorte entre le marteau (les résistances masculines) et l'enclume (le mérite), elles semblent s'en 
dépêtrer en évacuant l'idée qu'elles pourraient être personnellement discriminées:

Je n'ai jamais ressenti ça [être traitée autrement qu'un camarade], parce qu'ici il y a toujours eu des femmes élues. Et c'était même le cas bien avant cette dernière règle qu'est la Loi sur la parité. [Conseillère municipale, gauche (PCP), 35 ans, célibataire, sans enfant]

Inégalités de genre

La classe 2 représente $30 \%$ des UCE classées. Les discours qui ont le plus contribué à la formation de cette classe sont les réponses aux questions sur l'in/égalité et la discrimination. Ils proviennent surtout des femmes politiques plus âgées (54-78 ans) et de gauche.

Dans cette classe, nous avons identifié cinq thèmes: la discrimination que vivent les femmes en général, la discrimination que vivent les femmes politiques, la fragilisation des carrières politiques féminines, les différences entre hommes et femmes, en particulier dans leur façon de faire de la politique, et les réponses, en termes d'éducation ou de formation surtout, à donner aux inégalités de genre.

Le premier thème révèle que ces élues de gauche sont conscientes de l'existence des discriminations auxquelles l'ensemble des femmes sont confrontées:

Je pense que oui, les femmes en général sont victimes de discrimination. Oui, il suffit de regarder les statistiques et ça se voit. Elles sont victimes de discrimination dans l'accès aux postes de responsabilité, n'est-ce pas? [Députée d'une Assemblée municipale, gauche (BE), 44 ans, mariée, sans enfant]

Les femmes politiques sont également perçues comme un groupe discriminé, au même titre que "les femmes en général". Mais lorsque les interviewées dénoncent les inégalités de genre à l'œuvre dans le monde politique, elles n'évoquent pas qu'elles sont incluses dans cette catégorie. En d'autres termes, on est toujours ici dans le registre de la discrimination collective et non dans celui de la discrimination personnelle, dont on a vu auparavant (classe 1) qu'elle était minimisée:

Il n'y a pas autant de femmes que d'hommes dans la vie politique, cela signifie qu'il y a bien une discrimination. Le système politique est essentiellement auto-éligible, c'est-à-dire que les hommes sont choisis par eux-mêmes... ils sont proposés par eux-mêmes, c'est ainsi. [Conseillère municipale, gauche (PS), 65 ans, mariée, 3 enfants adultes]

Plusieurs comportements masculins fragilisent la carrière politique des femmes. En particulier, les plus âgées dénoncent le paternalisme de certains hommes politiques et l'irritabilité de ceux qui ne supportent pas qu'elles fassent carrière. Elles observent que les élues se sentent très visibles dans cet univers masculin et donc relativement vulnérables, devant alors être plus performantes que les hommes afin 
d'être reconnues. Dans ce contexte, les femmes font leur travail politique sous pression, d'autant plus quand les soutiens professionnels et/ou privés font défaut:

La femme politique doit démontrer plus de choses, elle est plus sous observation et plus visible. Elle est aussi plus fragile, parce que tout ça endommage sa vie personnelle. Parce qu'un homme politique est généralement soutenu par sa femme, tandis que le mari d'une femme politique, lui, s'associe moins avec elle, il la soutient moins. [Conseillère municipale, gauche (PS), 65 ans, mariée, 3 enfants adultes]

Un autre thème est centré sur la différence des sexes. Notamment, les femmes sont perçues comme plus sensibles, plus humaines et plus pacifiques que les hommes, et ces différences se reflètent dans leurs manières respectives de travailler en politique. Parfois, la complémentarité entre hommes et femmes associée à la différence des sexes est valorisée, mais surtout, les élues valorisent les qualités spécifiquement féminines et espèrent que celles-ci seront le support d'un changement des pratiques en vigueur dans le monde politique masculin:

Quand une femme est dans une position de leader, elle est meilleure qu'un homme. Quand elle est bonne, elle est vraiment bonne... enfin, je veux dire, par comparaison. [Députée d'une Assemblée communale, gauche (BE), 54 ans, célibataire, sans enfant]

Le dernier thème est l'éducation à l'égalité. Du point de vue des interviewées, les inégalités entre femmes et hommes en général et, plus spécifiquement, la sous-représentation des femmes en politique, reposent en bonne partie sur la socialisation différentielle des sexes. Dans cette perspective, l'intégration de valeurs égalitaires dans les politiques d'éducation et de formation leur paraît un bon moyen d'améliorer le destin des femmes:

Les femmes sont de meilleures leaders que les hommes, c'est prouvé. [Alors, pourquoi n'arrivent-elles pas aux postes de pouvoir?] Je ne sais pas. Je ne comprends pas. Ça ne peut être qu'une question de société, ça passe sous une forme soft dans l'éducation. [Députée d'une Assemblée communale, gauche (BE), 54 ans, célibataire, sans enfant]

\section{Conceptions de la profession politique}

La classe 3 représente $22 \%$ des UCE classées. Elle est formée par les discours sur la compétence politique et, plus modérément, sur la mobilisation contre les inégalités de genre. Ils émanent davantage des militantes âgées de 32 à 53 ans, indépendamment de leur appartenance politique. Nous avons identifié quatre thèmes: les compétences nécessaires en politique, les qualités personnelles, la reconnaissance des pairs et la mobilisation pour l'égalité.

Les discours montrent que le spectre des compétences nécessaires au travail politique est très étendu. La personne militante doit savoir communiquer, écouter, observer, réfléchir, être disponible, honnête, humaine, humble, intelligente, in/formée, 
compétente, travailleuse, avoir du bon sens, aimer la communauté, connaître son entourage, être personnellement désintéressée, avoir de l'intérêt pour la cause publique et s'y dévouer, servir le bien commun, défendre ses idéaux, avoir le sens de la justice, une bonne capacité de travail et savoir prendre des décisions:

Les caractéristiques importantes pour faire de la politique, ce sont le travail, la capacité de travail, l'honnêteté, la compétence, l'écoute, et savoir communiquer. [Présidente d'une commune, gauche (PCP), 32 ans, mariée, 1 enfant petit]

Outre les compétences politiques, les qualités personnelles sont des outils importants pour s'investir en politique. Pour expliquer leur engagement et à la fois rendre compte de leur manière de militer, les jeunes élues mettent en avant leurs aptitudes personnelles, se décrivant comme des personnes équilibrées, engagées, créatives et avec un certain sens de l'humour, solidaires et ambitieuses:

En tant que personne, en tant que femme politique, en tant qu'étudiante, je suis quelqu'un qui a de l'ambition, mais pas une ambition démesurée. J'ai une ambition personnelle. Je suis une personne solidaire et très équilibrée. [Députée d'une Assemblée municipale, gauche (PS), 33 ans, célibataire, sans enfant]

Cette conception exigeante de la profession politique, qui multiplie les compétences politiques et les qualités personnelles attendues de l'élue, semble être le garant de la reconnaissance par les pairs. Ainsi, sur un plan individuel, les interviewées s'estiment généralement bien intégrées, respectées et non stigmatisées:

Mes collègues du conseil municipal me respectent. Personne ne dit: "elle fait ça parce que c'est une femme". Non, d'aucune façon. Au contraire, je sens un respect. Je dirais même, sans fausse modestie, que certaines personnes ont de l'admiration pour moi. Oui, c'est un fait. [Conseillère municipale, droite (PSD), 45 ans, mariée, 2 enfants petit et adolescent]

Le dernier thème est centré sur la mobilisation contre les inégalités de genre. Dans leur parcours, toutes les interviewées ont déjà milité pour l'égalité, d'une façon ou $\mathrm{d}^{\prime}$ une autre, à travers un parti, une association ou un mouvement de femmes:

Oui, j'ai participé à plusieurs actions collectives [pour l'égalité] et à des initiatives locales de la municipalité, ainsi que du MDM [Mouvement démocratique des femmes]. [Conseillère municipale, gauche (PCP), 35 ans, célibataire, sans enfant]

\section{Maternité et mariage comme obstacle au travail politique}

La classe 4 représente 17\% des UCE classées. Elle fait partie du deuxième groupe thématique émergeant de la classification descendante hiérarchique effectuée par Alceste. Les discours qui ont le plus contribué à la formation de cette classe sont liés à la conciliation, résultant surtout des plus jeunes élues qui ont des enfants et sont 
mariées. La "conciliation" entre le travail politique et la vie familiale est structurée autour de trois thèmes: le travail domestique en tant que frein aux carrières politiques, les compétences organisationnelles des mères et l'indispensable soutien du conjoint.

Le travail domestique, incluant les tâches ménagères, la prise en charge des enfants et le soin aux parents, est un frein à la carrière politique des interviewées. Pour ces femmes, le quotidien domestique représente une lourde charge de travail, qui rend ladite conciliation difficile et conditionne leur investissement politique. Dans ce contexte, elles tendent à déléguer une partie de ce travail à des membres de la famille, à une femme de ménage ou à une crèche. Mais lorsqu'un de ces soutiens devient défaillant, l'articulation de leurs diverses activités se fragilise. L'idée est même émise qu'une femme engagée en politique aurait intérêt à ne pas avoir d'enfant. Le militantisme, qui ne prévoit ni horaire ni week-end, se conjugue donc difficilement avec une vie familiale qui elle aussi exige beaucoup de disponibilité:

J'ai une personne à la maison tous les jours. Elle s'occupe des tâches ménagères, du nettoyage de la maison, de la lessive et elle m'aide avec les repas. Ce serait impossible sans cette aide, car en fait, mon mari n'a pas non plus d'horaire pour rentrer à la maison le soir, et s'il fallait, au moment où il rentre, avoir à faire le dîner ou devoir sortir pour l'acheter, cela n'aurait aucun sens. [Présidente d'une commune, gauche (PCP), 32 ans, mariée, 1 enfant petit]

Les conditions dans lesquelles le travail domestique se fait étant un élément fondamental de la possibilité même du militantisme des femmes mariées, ce travail leur demande une organisation rigoureuse, où rien n'est laissé au hasard. Elles font ainsi valoir leurs compétences organisationnelles comme un facteur d'ajustement important entre le temps familial et le temps militant:

Le secret, c'est de bien rentabiliser le temps. Je dis toujours: la journée a 24 heures pour tout le monde. Je ne perds pas de temps, je n'ai pas de temps mort. C'est l'organisation qui est fondamentale. Toutes mes minutes sont importantes, très importantes. [Conseillère municipale, droite (PSD), 45 ans, mariée, 2 enfants petit et adolescent]

Le troisième thème sur le militantisme des femmes mariées est le soutien des conjoints au travail domestique, et donc au travail politique de leur compagne. Les discours des élues, tant de gauche que de droite, montrent que ce soutien masculin ne va pas de soi et qu'elles doivent lutter pour obtenir que les tâches ménagères et éducatives soient partagées au sein du couple. La contribution domestique de leur conjoint détermine les modalités de leur engagement politique:

À la maison, rien n'a jamais été partagé. La responsabilité m’est toujours revenue, particulièrement au niveau logistique. C'est évident par exemple avec mon fils. Et cela m'a empêchée de faire de nombreux choix [notamment au niveau politique]. [Conseillère municipale, droite (PSD), 57 ans, mariée, 1 enfant adulte] 


\section{Stratégies des femmes tokens face au sexisme}

La classe 5 représente $21 \%$ des UCE classées. Les discours qui ont le plus contribué à sa formation résultent avant tout des dynamiques du tokenism, de la mobilisation et de la conciliation. Ils émanent principalement des élues de droite qui ont entre 54 et 78 ans. Nous identifions deux thèmes qui ont trait au sexisme qu'elles rencontrent à partir de leur double statut minoritaire, donc en tant que femmes et en tant que tokens: leurs stratégies face aux agressions des dominants et le sexisme ordinaire des univers professionnels masculins.

Tout au long de leur parcours, les élues plus âgées se sont fait régulièrement rappeler par les hommes qu'elles sont d'abord des femmes, objets de conquête de ceux-ci. Dans tous les espaces de vie (dans la rue, dans l'entreprise et dans le monde politique), ces femmes ont fait l'expérience de regards masculins qui s'expriment avec plus ou moins de violence, allant de tentatives de séduction déplacées à des agressions et du harcèlement sexuel. Pour résister à ces rappels à l'ordre sexué et se défendre des agressions, elles adoptent toutes sortes de stratégies. Notamment, elles utilisent l'humour, cherchent à décourager leur agresseur en lui montrant qu'elles n'ont pas peur de lui, feignent de l'ignorer pour éviter que la situation dégénère, et parfois lui opposent une riposte frontale:

Quand je suis confrontée à une situation de discrimination, je suis frontale. Quand je vois quelque chose que je n'aime pas, c'est quelque chose qui... je suis très... impulsive! Vous voyez? Moi, devant une telle situation, je réagis à chaud, ce qui, parfois, n'est pas très bon. [Présidente d'une commune, droite (PSD), 53 ans, mariée, sans enfant].

Dans les partis de droite, les discours des élues mettent également en avant qu'elles sont ou ont été renvoyées à leur statut de femme par des comportements plus subtils, qui relèvent d'un sexisme ordinaire et routinier. Ainsi, dans leur quotidien politique ou professionnel, il leur est arrivé plus d'une fois de se voir dénié, par les hommes, le droit à la parole, de disparaître derrière l'usage généralisé du masculin ou d'être tenues à l'écart des informations:

Un jour dans l'entreprise où je travaillais, l'un des directeurs a dit à la fin d'une réunion: "J'ai encore deux ou trois questions, mais on verra ça plus tard." Le président lui dit: “Comment ça, plus tard?" Le directeur, jetant un œil dans ma direction, lui a répondu: "J'en parlerai avec vous plus tard." Le président lui a dit: "Non, les choses de l'entreprise, nous devons en parler ici." "Pas possible en sa présence" semblait penser le directeur en se tournant de nouveau vers moi [Conseillère municipale, droite (PSD), 57 ans, mariée, 1 enfant adulte]

\section{Discussion}

Nous proposons de discuter les dynamiques de genre qui œuvrent dans le contexte du tokenism en distinguant celles qui relèvent de stratégies de contestation de 
l'ordre social sexué par les femmes politiques et celles qui contribuent à le maintenir, incluant aussi bien les discriminations exercées par le groupe dominant que certaines des stratégies adoptées par ces femmes. En particulier, cette discussion des résultats sera attentive à l'ancrage social (âge, statut familial et appartenance politique) des dynamiques observées, car même si la plupart des interviewées ont contribué à la formation de chaque classe thématique, leurs représentations varient parfois fortement selon leurs positions sociales et politiques.

Globalement tout d'abord, les résultats montrent que les élues ne se considèrent pas comme des femmes-alibis, qui seraient instrumentalisées par les hommes et les partis pour ne rien changer au système politique antérieur à l'introduction des quotas. En effet, elles ont une vision positive d'elles-mêmes (compétences politiques et qualités personnelles) et se sentent reconnues dans leur travail politique (classe 3), et elles s'estiment généralement bien intégrées, respectées et non discriminées sur un plan personnel (classe 1). Pourtant, les discriminations que vivent "les femmes en général" et "les femmes politiques" en particulier ne font pas de doute pour elles (classe 2). Certaines dénoncent par exemple le paternalisme des hommes politiques et le prix à payer pour que les élues se fassent une place dans le monde masculin qu'elles ont choisi d'investir: en termes de compétences, les exigences sont beaucoup plus élevées à leur égard qu'à celui des hommes (classe 2). En d'autres termes, une double dynamique paraît caractériser ce contexte marqué par le genre: les carrières politiques des femmes sont freinées par les comportements de domination qu'exercent les hommes tant dans la sphère publique (e.g., dénier le droit de parole aux élues, classe 5) que privée (e.g., ne pas faire sa part de travail domestique, classe 4), mais ce qui vaut pour les autres ne vaut pas forcément pour soi, puisque les femmes qui reconnaissent ce mécanisme de discrimination collective (envers les femmes en général et les femmes politiques) ne s'estiment pas toujours concernées. La minimisation de la discrimination personnelle, doublée de la reconnaissance de la discrimination collective, serait donc un moyen de se démarquer de son groupe d'appartenance dominé - les femmes - afin de légitimer sa place dans le groupe dominant - les hommes. En ce sens, la minimisation de la discrimination personnelle peut favoriser la mobilité de quelques individus minoritaires mais n'aide pas à franchir les barrières intergroupes et donc à rehausser le statut du groupe d'appartenance initial lorsque celui-ci occupe une position sociale dominée.

Ces dynamiques de genre se différencient systématiquement selon les groupes d'âge, et parfois selon les positions politiques. Dans notre étude, ce sont d'abord les élues de gauche les plus jeunes (32-53 ans) qui s'estiment non stigmatisées et non discriminées à un niveau personnel, et ce sont les interviewées plus âgées (54-78 ans) qui dénoncent davantage les discriminations dont les femmes, les femmes politiques et elles-mêmes sont ou ont été victimes, qu'elles soient de gauche (classe 3) ou de droite (classe 5). Par exemple, les élues de gauche plus âgées déplorent qu'une femme politique doive travailler deux fois plus qu'un homme politique (classe 3) et les élues de droite plus âgées elles aussi s'insurgent contre les agressions ou le harcèlement sexuel qu'elles ont eu à subir (classe 5). Cette différence de perception selon les groupes d'âge peut s'expliquer par le fait que 
l'expérience de la discrimination augmente avec le temps, donc avec l'âge. Le cumul des inégalités et des contraintes vécues tout au long du parcours de vie conduirait les élues plus âgées à prendre la mesure de la stabilité de la hiérarchie des sexes, fortes des expériences de résistance qu'elles ont déployée par toutes sortes de stratégies - mépris, persuasion, coups, humour - pour se défendre aussi bien du sexisme ordinaire dans l'hémicycle politique que des violences sexuelles vécues en entreprise ou dans la rue (classe 5). Pour leur part, si les femmes politiques plus jeunes, surtout dans les partis de gauche, se sentent moins discriminées, c'est peut-être parce que l'égalité des sexes a réellement progressé dans le monde politique ou du moins dans leur parti. Si tel est le cas, leur évaluation de la discrimination personnelle n'est pas du déni. Mais il est aussi possible que leur place dans le groupe dominant soit encore plus difficile à consolider que celle des élues plus âgées, parce que leur entrée en politique a eu lieu, en moyenne, 10 ans plus tard que leurs aînées. Le cas échéant, la sous-estimation du sexisme de leurs camarades masculins serait alors un moyen de poursuivre leur carrière, en ne se laissant pas enfermer dans un statut de "femme dominée". Cette volonté de sortir d'un statut inférieur est fort bien illustrée par la manière dont les plus jeunes interviewées, indépendamment de leur appartenance politique, conçoivent leur métier: l'élue doit cumuler toutes les qualités et toutes les compétences possibles et imaginables (classe 3) et doit faire une double journée de travail ou tout au moins assurer ladite conciliation de ses engagements politiques et familiaux, aussi bien envers ses enfants et ses parents qu'envers son conjoint dont le soutien lui fait trop souvent défaut (classe 4). Il faut relever que ce manque de soutien est déploré par les militantes de droite comme de gauche: la division sexuelle du travail au sein des couples transcende les oppositions idéologiques.

Un autre résultat appuie l'idée que les plus jeunes cherchent à contrer leur assignation à un groupe d'appartenance dominé en profilant une sorte de superwoman capable de tout gérer à la fois: leur attachement au principe du mérite (classe 1). Même favorables à la Loi sur la parité, elles subordonnent sa mise en œuvre au respect et à la reconnaissance des mérites individuels, en particulier celles qui militent au PS. Ce faisant, elles prennent un risque, car elles savent que ce sont toujours le mérite et les compétences des femmes qui sont questionnés, rarement le mérite et les compétences des hommes. Mais elles observent aussi les stratégies que les hommes politiques et les partis, y compris le leur, qui est à l'origine de la mise en place des quotas au Portugal, déploient pour ne pas appliquer la Loi sur la parité, détournant de multiples manières l'objectif d'égalité qu'elle est censée concrétiser (classe 1). Du coup, dans ce contexte ambivalent où l'égalité est censée s'accommoder du maintien des privilèges des hommes, l'adhésion à l'idéologie méritocratique paraît relever d'une stratégie que les jeunes élues de gauche mobilisent pour forcer les partis à repenser leur conception de l'égalité des sexes et pour mener à bien leur carrière malgré leur position de tokens. Peut-être même est-ce là une stratégie visant à faire oublier qu'elles sont des femmes, un objectif qui intéresserait nettement moins les interviewées plus âgées.

En effet, ces dernières sont particulièrement attentives, surtout dans les partis de gauche, à la différence des sexes et valorisent des qualités qu'elles jugent 
spécifiquement féminines (e.g., le pacifisme, classe 2). Adoptant d'autres modes de travail que les hommes politiques, elles estiment que les femmes, pour autant qu'elles soient féministes, pourraient rendre plus égalitaires le fonctionnement des partis et la nature même de la politique (classe 2). Dans cette perspective, leurs stratégies d'action prioritaires visent à changer les mentalités, à travers des politiques d'éducation et de formation qui devraient être aptes à contrer la socialisation différentielle des sexes toujours dominante aujourd'hui. Les plus jeunes pour leur part évoquent rarement ce type de politiques et se centrent davantage sur ce qu'elles peuvent faire depuis l'intérieur du parti ou d'une association pour lutter contre les inégalités de genre (classe 3).

Notre étude a montré que l'application de la Loi sur la parité au Portugal ne bouleverse pas l'ordre de genre structurant la politique locale et va dans le même sens que les travaux cités en introduction qui ont été menés dans d'autres domaines professionnels: la hiérarchie des sexes ne disparaît pas dans un contexte de tokenism, la division sexuelle du travail qu'elle produit continue à organiser tant la sphère politique que la sphère privée. Les élues que nous avons interrogées reconnaissent ces discriminations que les femmes en général et les femmes politiques en particulier ont à affronter, mais les stratégies qu'elles développent pour y faire face pourront-elles, à terme, favoriser une égalité de fait entre les sexes? Par exemple, le modèle de la superwoman ne risque-t-il pas de renforcer le niveau de compétences exigé d'elles, souvent nettement plus élevé que celui attendu des hommes? Valoriser les qualités dites féminines ne risque-t-il pas de consolider la différence des sexes qui sert de support à la justification des inégalités? De même, la valorisation des mérites individuels et la minimisation de la discrimination personnelle pourraient être récupérées pour dénier la domination des hommes et la subordination des femmes. Certes, avec de telles stratégies, parfois différentes selon leur appartenance politique et selon l'étendue de leur parcours politique, les interviewées tentent bien de combattre les inégalités de genre, mais notre étude a montré qu'elles le font en mettant à distance leur double statut minoritaire de femme et de token, pour privilégier davantage un statut qui semble leur garantir une relative légitimité: celui d'individu politique comme les autres, capable d'intégrer un monde d'hommes au même titre que n'importe lequel d'entre eux. Or, penser de manière égale des individus qui ne le sont pas dans la réalité sociale peut créer l'illusion d'échapper à l'ordre de genre mais ne permet certainement pas de le transformer.

\section{Références}

Amâncio, Lígia (2007), “Género e divisão do trabalho doméstico: o caso português em perspectiva", in Karin Wall et Lígia Amâncio (dirs.), Família e Género em Portugal e na Europa. Atitudes Sociais dos Portugueses, Lisbonne, Imprensa de Ciências Sociais, pp. 181-210.

Apfelbaum, Erika (1999), "Relations of domination and movements for liberation: An analysis of power between groups (Abridged)", Feminism \& Psychology, 9 (3), pp. 267-272, DOI: 10.1177/0959353599009003003 
Barreto, Manuela, Naomi Ellemers, et Maria Soledad Palacios (2004), "The backlash of token mobility: The impact of past group experiences on individual ambition and effort", Personality and Social Psychology Bulletin, 30 (11), pp. 1433-1445, DOI: $10.1177 / 0146167204264336$

Bereni, Laure, Catherine Marry, Sophie Pochic, et Anne Revillard (2011), “Le plafond de verre dans les ministères: Regards croisés de la sociologie du travail et de la science politique", Politiques et Management Public, 28, pp. 139-156, DOI: $10.4000 / \mathrm{pmp} .4141$

Cognard-Black, Andrew (2004), “Will they stay, or will they go? Sex-atypical work among token men who teach", The Sociological Quarterly, 45 (1), pp. 113-139.

Crosby, Faye (1984), "The denial of personal discrimination”, American Behavioral Scientist, 27 (3), pp. 371-386, DOI: 10.1177/000276484027003008

Crosby, Faye, et Susan Clayton (2001), “Affirmative action: Psychological contributions to policy", Analyses of Social Issues and Public Policy, 1 (1), pp. 71-87, DOI: $10.1111 / 1530-2415.00004$

Dahlerup, Drude (2008), “Gender quotas: Controversial but trendy", International Feminist Journal of Politics, 10 (3), pp. 322-328, DOI: 10.1080/14616740802185643

Ellemers, Naomi, Russell Spears, et Bertjan Doosje (1997), “Sticking together or falling apart: Ingroup identification as a psychological determinant of group commitment versus individual mobility", Journal of Personality and Social Psychology, 72 (3), pp. 617-626, DOI: http://dx.doi.org/10.1037/0022-3514.72.3.617

Fassa, Farinaz, et Sabine Kradolfer (2010), Le plafond de fer de l'université. Femmes et carrières, Zürich, Seismo.

Floge, Liliane, et Deborah Merrill (1986), “Tokenism reconsidered: Male nurses and female physicians in a hospital setting", Social Forces, 64 (4), pp. 925-947.

Gaspard, Françoise (2007), "Féminisation de la politique? Un regard international", Travail, Genre et Sociétés, 2 (18), pp. 135-138.

Gaspard, Françoise, Claude Servan-Schreiber, et Anne Le Gall (1992), La représentation paritaire. Au pouvoir citoyennes: liberté, égalité, parité, Paris, Seuil.

Geist, Claudia (2010), “Men's and women's reports about housework”, in Judith Treas et Sonja Drobnic (dirs.), Dividing Domestic. Men, Women and Household Work in Cross-National Perspective, Stanford, CA, Stanford University Press, pp. 217-240.

Heikes, J. (1991). "When men are the minority: The case of men in nursing”, The Sociological Quarterly, 32 (3), pp. 389-401.

Heilman, Madeline, Aaron Wallen, Daniella Fuchs, et Melinda Tamkins (2004), "Penalties for success: Reactions to women who succeed at male tasks", Journal of Applied Psychology, 89 (3), pp. 416-427, DOI: http://dx.doi.org/10.1037/0021-9010.89.3.416

Kanter, Moss Rosabeth (1993 [1977]), Men and Women of the Corporation, New York, Basic Books (2ème édition).

Krook, Mona Lena, et Diana O’Brien (2010), “The politics of group representation: Quotas for women and minorities worldwide", Comparative Politics, 42 (3), pp. 253-272, DOI: http://dx.doi.org/10.5129/001041510X12911363509639

Lépinard, Eléonore (2007), L'égalité introuvable: la parité, les féministes et la République, Paris, Presses de Sciences po. 
Ott, Marlies (1989), "Effects of the male-female ratio at work: Policewomen and male nurses", Psychology of Women Quarterly, 13 (1), pp. 41-57, DOI: 10.1111/j.1471.

Roux, Patricia, avec la collaboration de Valérie Perrin, Marianne Modak, et Bernard Voutat (1999), Couple et égalité. Un ménage impossible, Lausanne, Réalités Sociales.

Santos, Maria Helena (2011), Do Défice de Cidadania à Paridade Política. Testemunhos de Deputadas e Deputados, Porto, Afrontamento.

Santos, Maria Helena, et Lígia Amâncio (2012), “Género e política: análise sobre as resistências nos discursos e nas práticas sociais face à lei da paridade", Sociologia, Problemas e Práticas, 68, pp. 79-101, DOI: 10.7458/SPP201268694

Santos, Maria Helena, Lígia Amâncio, et Hélder Alves (2013), “Gender and politics: The relevance of gender on judgements about the merit of candidates and the fairness of quotas", Portuguese Journal of Social Science, 12 (2), pp. 133-149, DOI: 10.1386/pjss.12.2.133_1

Santos, Maria Helena, et Lígia Amâncio (2014), “Sobreminorias em profissões marcadas pelo género: consequências e reações", Análise Social, XLIX (3), pp. 700-726.

Santos, Maria Helena, Lígia Amâncio, et Patricia Roux (2015), “Numbers do not tell the whole story: Gender and medicine in Portugal, Women's Studies International Forum, 53, pp. 73-82, DOI: 10.1016/j.wsif.2015.08.005.

Scott, Joan (2005), Parité! L'universel et la différence des sexes, Paris, Albin Michel.

Silva, Manuel Carlos, Ana Reis Jorge, et Aleksandra Queiroz (2012), “Divisão sexual do trabalho doméstico: entre representações e práticas", Configurações, 9, pp. 135-159: http://configuracoes.revues.org/1158

Sineau, Mariette (2011), Femmes et pouvoir sous la Cinquième République. De l'exclusion à l'entrée dans la course présidentielle, Paris, Presses de Sciences po (2 $2^{\mathrm{ème}}$ édition remaniée et augmentée).

Staerklé, Christian, Christophe Delay, Lavinia Gianettoni, et Patricia Roux (2007), Qui a droit à quoi? Représentations et légitimation de l'ordre social, Grenoble, Presses Universitaires de Grenoble.

Taylor, Donald, et David Mckirnan (1984), “Theoretical contributions: A five-stage model of intergroup relations", British Journal of Social Psychology, 23 (4), pp. 291-300, DOI: 10.1111/j.2044-8309.1984.tb00644.x

Wall, Karin, et Maria das Dores Guerreiro (2005), "A divisão familiar do trabalho", in Karin Wall (dir.), Famílias em Portugal. Percursos, Interacções e Redes Sociais, Lisbonne, Imprensa das Ciências Sociais, pp. 303-362.

Williams, Christine (1995), Still a Man's World. Men Who Do "Women's Work", Londres, University of California Press.

Wright, Stephen (2001), “Restricted intergroup boundaries: Tokenism, ambiguity and the tolerance of injustice", in John Jost et Brenda Major (dirs.), The Psychology of Legitimacy. Emerging Perspectives on Ideology, Justice, and Intergroup Relations, New York, Cambridge University Press, pp. 223-264.

Yoder, Janice (1994), “Looking beyond numbers: the effects of gender status, job prestige, and occupational gender-typing on tokenism processes", Social Psychology Quarterly, 57 (2), pp. 150-159.

Young, Iris Marion (1990), Justice and the Politics of Difference, Princeton, Princeton University Press. 
Maria Helena Santos (corresponding author). Psychologue sociale, investigadora de pós-doutoramento à l'Instituto Universitário de Lisboa (ISCTE-IUL) (CIS-IUL), Lisbonne, Portugal, et Centre en Etudes Genre LIEGE, Université de Lausanne, Lausanne, Suisse. E-mail: mhelena.rc.santos@gmail.com

Patrícia Roux. Ancienne professeure en Etudes Genre à l'Université de Lausanne et co-rédactrice en chef de la revue Nouvelles Questions Féministes, Lausanne, Suisse. E-mail: patricia.roux@citycable.ch

Lígia Amâncio. Psychologue sociale et professeure à l'Instituto Universitário de Lisboa (ISCTE-IUL) où elle dirige le Centre de Recherche en Psychologie (CIS-IUL), Lisbonne, Portugal. E-mail: ligia.bq.amancio@gmail.com 
